\title{
The Effect of Combination of Red, Infrared and Blue Wavelengths of Low-Level Laser on Reduction of Abdominal Girth: A Before-After Case Series
}

\author{
Katayoon Montazeri ${ }^{{ }^{*}}$, Soheila Mokmeli ${ }^{2}$, Maryam Barati ${ }^{3}$ \\ ${ }^{1}$ Laboratory for Auditory Neuroscience, ENT and Head \& Neck Research Centre, Iran University of Medical Sciences, Tehran, \\ Iran \\ ${ }^{2}$ Canadian Optic and Laser Center (COL Center), Research Unit, Victoria BC Canada \\ ${ }^{3}$ Laser Application in Medical Sciences Research Center (LAMSRC), Shahid Beheshti University of Medical Sciences, Tehran, \\ Iran
}

\author{
*Correspondence to \\ Katayoon Montazeri, \\ M.D.Laboratory for Auditory \\ Neuroscience, ENT and Head \\ \& Neck Research Centre, Iran \\ University of Medical Sciences, \\ Tehran, Iran. \\ Tel:+98 2166504294; \\ Fax:+98 2166525329. \\ Email:katayoon_mon@yahoo.com
}

Published online 29 August 2017

\begin{abstract}
Introduction: The objective of this study was to assess the efficacy of a combination of 3 wavelengths (including red, infra-red, and blue) of low-level laser (LLL) as a non-invasive therapeutic method to reduce abdominal girth. To achieve biochemical activity on adipocytes, a red laser was used in our selective laser combination. Near-infrared laser was used to increase depth of penetration. Nitrosyl complexes of hemoglobin ( $\mathrm{NO}-\mathrm{Hb})$ are sensitive to blue light, thereby leading to increase in release of biologically active nitric oxide (NO), which can affect tissue perfusion. Thus, a blue LED was added to the laser combination.

Methods: Eighteen females participated in the study. Twelve sessions of laser therapy were performed, 2 sessions per week for each subject. Continuous wave diode lasers, including red $(630 \mathrm{~nm})$, infra-red $(808 \mathrm{~nm})$, and a blue LED $(450 \mathrm{~nm})$ were applied and were all designed by the Canadian Optic and Laser Center.

Results: Statistical analyses revealed that upper abdomen size significantly decreased from pre- $(91.86 \pm 11.16)$ to post- $(87.41 \pm 10.52)$ low-level laser therapy (LLLT) $(P<0.001)$. Middle abdomen size showed significant reduction from pre- $(97.02 \pm 8.82)$ to post- $(91.97 \pm 8.49)$ LLLT $(P<0.001)$. Lower abdomen size significantly decreased from pre- $(100.36 \pm 9.45)$ to post$(95.80 \pm 8.52)$ LLLT $(P<0.001)$.

Conclusion: Based on this case series pilot investigation, the combination of 3 different wavelengths of LLL was effective for abdominal girth reduction in $100 \%$ of our subjects $(P<0.001)$, without any side effects. Future studies will assess the long-term benefits of this laser combination for reduction of subcutaneous fat deposits.

Keywords: Diode lasers; Low-level laser therapy; Non-invasive girth reduction.
\end{abstract}

\begin{abstract}
Introduction
The term cellulite (also known as gynoid lipodystrophy and adiposis edematosa) refers to the herniation of subcutaneous fat within fibrous connective tissue that manifests as skin uneven dimpling or "orange peel" appearance..$^{1-3}$ Cellulite is more common on the buttocks, lower limbs and abdomen. ${ }^{1,2}$ Cellulite affects mainly women as a result of "differences in the structural anatomy of subcutaneous tissue in women, and the possible influence of estrogen."' The prevalence of cellulite in post-adolescent females was estimated to be between $80 \%$ and $90 \%, 3,5$ which led to the development of cosmetic complications. Cellulite appears to be a "multifactorial disorder" which is likely due to metabolic alterations, changes in lymphatic and vascular microcirculation, dieting too hard or too much, sex-specific dimorphic,
\end{abstract}

skin architecture, alteration of connective tissue structure, and hormonal and genetic factors. ${ }^{5-7}$ Emanuele et $\mathrm{al}^{3}$ traced "the genetic component of cellulite to particular polymorphisms in the angiotensin converting enzyme (ACE) and hypoxia-inducible factor 1A (HIF1a) genes." There are various modalities in the treatment of localized subcutaneous fat deposits (massage or mechanical manipulation, mesotherapy, bipolar radiofrequency, liposuction, laser lipolysis, etc), but empirical evidence for the efficacy of these strategies is limited. ${ }^{3}$

Low-level laser therapy (LLLT) is a non-thermal, noninvasive technique which is practiced by physicians and laser therapists for a wide variety of diseases. It is used for tissue regeneration and bio-stimulation, pain reduction, swelling, inflammatory response in orthopedic injuries and degenerative diseases, wound healing and numerous

Please cite this article as follows: Montazeri K, Mokmeli S, Barati M. The effect of combination of red, infrared and blue wavelengths of low-level laser on reduction of abdominal girth: a before - after case series. J Lasers Med Sci. 2017;8(Suppl 1):S22-S26. doi:10.15171/ jlms.2017.s5. 
cosmetic procedures such as breast augmentation and lipoplasty. ${ }^{8-10}$ It is used as an adjunct to liposuction to reduce pain and inflammation, ${ }^{11}$ and it has also been shown to be effective for non-invasive body contouring. Reduction in cholesterol and leptin levels are other significant clinical benefits of LLLT which were reported in several studies. ${ }^{11,12}$

US Food and Drug Administration (FDA) approved the clinically tested LLL ZERONA" device as a "non-invasive dermatological aesthetic treatment for the circumferential reduction of hips, waist, and thighs."

Laser irradiation mechanism of action on non-invasive body contouring and cellulite reduction remains somewhat controversial. LLLT was suggested to induce the formation of transitory micro-pores in the cell membrane of adipocytes, allowing intracellular lipids to leak out. ${ }^{13-16}$ Though, the biochemical mechanism of action of LLLT on adipose tissue is still controversial, one possible explanation might be that, absorption of laser light by mitochondrial chromophores (particularly, cytochrome $\mathrm{C}$ oxidase) of the adipocytes leads to increase of cyclic adenosine monophosphate (cAMP) production. Elevated cAMP stimulates cytoplasmic lipase, an enzyme that converts triglycerides into fatty acids and glycerol which can both pass through transitory pores, thereby leading to shrinkage of the adipocytes. ${ }^{13-15}$

The ability of LLL for non-invasive body contouring has been investigated by many researchers. Lach and Pap conducted another study among 25 subjects who underwent a series of treatments with infrared and red lasers, followed by massage. In total, 14 subjects showed a $5 \%$ to $35 \%$ reduction in fat thickness and improvement in the appearance of cellulite in the thighs at the end of the treatment period. ${ }^{17}$

A review of the studies which applied LLLT for body contouring revealed that some researchers used red laser alone, ${ }^{18-22}$ and others used a combination of red and infrared lasers. ${ }^{17,23}$

In this study, a combination of 3 different wavelengths of diode lasers was used, and the effects of this combination were investigated on abdominal girth reduction.

Lasers were selected according to the findings of several researches, as subsequently discussed.

Neira et al investigated the action of red laser $(635 \mathrm{~nm}, 10$ $\mathrm{mW}$ intensity) on human adipocytes taken from lipectomy samples. Transmission electron microscope (TEM) and scanning electron microscope (SEM) images revealed the formation of transient micro-pores in cell membrane of the adipocytes. Subsequently, up to $99 \%$ of fat could be released from the adipocytes, leading to complete deflation of adipocyte. ${ }^{23,24}$ Another proposed mechanism of action is based upon activation of complement cascade, which could induce adipocyte apoptosis leading to release of lipids through transient pores. ${ }^{10}$ A red $630 \mathrm{~nm}$ laser was used in our selective lasers combination to achieve this biochemical activity on adipocytes.
It was shown by several studies that penetration depth is significantly affected by wavelength. Longer wavelengths will penetrate further because both scattering and absorption of light are higher in shorter wavelengths. ${ }^{25-28}$ There is a so-called therapeutic window (also known as optical window) which defines the range of wavelengths from 650 to $1350 \mathrm{~nm}$, where effective tissue penetration of light is maximiz). ${ }^{28}$ A near-infrared laser $(808 \mathrm{~nm})$ was used to affect deeper fat layers.

On the other hand, Furchgot and Ehrreich demonstrated the ability of laser light to facilitate the release of nitric oxide (NO) from nitrosyl complexes of hemoglobin. Biologically active NO can stimulate vasodilation through the effect of NO on cGMP, followed by increased blood circulation in the tissue. ${ }^{29}$ Recently, it has been shown that the most effective wavelengths on light mediated release of NO are UV-A (320 to $400 \mathrm{~nm}$ ) and blue range (400 to $460 \mathrm{~nm}) \cdot{ }^{30-33}$ One of the cellulite predisposing factors is circulatory insufficiency. ${ }^{1,5-7}$ Over this fact, ameliorating tissue perfusion by blue light may be effective in decreasing problems of circulation and reducing localized adiposity. The experimental objective of this study, therefore, was to evaluate the application of a $630 \mathrm{~nm}$ red laser, $808 \mathrm{~nm}$ infra-red laser and a $450 \mathrm{~nm}$ blue LED (blue laser was not available) for non-invasive reduction of circumference in patients with abdominal localized adiposity.

\section{Methods}

This study was designed as a before-after case series. Eighteen adult females aged between 26 and 62 years with abdominal localized adiposity received 12 biweekly treatments (mean age \pm standard deviation [SD]: $44.61 \pm$ 10.98).

Subjects were selected according to the following exclusion and inclusion criteria:

Inclusion criteria comprised female gender patients who did not respond to diet and exercise, willingness to abstain from participating in any other treatment procedures for weight loss and girth reduction during the course of study, willing and able to maintain normal pre-study diet and exercise, and age between 20 and 65 years.

Exclusion criteria included body mass index (BMI) of 30 $\mathrm{kg} / \mathrm{m}^{2}$ or above, diabetes mellitus, cardiovascular disease, prior surgical interventions for body contouring or weight loss, current use of medication(s) known to affect weight levels and/or cause bloating or swelling, pregnancy, breast feeding and serious mental health illness.

This study was conducted in our private medical laser clinic, Tehran, Iran, between April 2013 and January 2014. Laser devices used included Red laser designed by Canadian optic and laser (COL) Company, 630 nm, 100 $\mathrm{mW}$, spot size $=1 \mathrm{~cm}^{2}$; continuous mode, power density $=0.1 \mathrm{~W} / \mathrm{cm}^{2}$; Infra-red laser designed by COL Company, $808 \mathrm{~nm}, 100 \mathrm{~mW}$, spot size $=1 \mathrm{~cm}^{2}$; continuous mode, power density $=0.1 \mathrm{~W} / \mathrm{cm}^{2}$; Blue LED designed by COL Company, $450 \mathrm{~nm}, 3000 \mathrm{~mW}$, spot size $=1 \mathrm{~cm}^{2}$. 
The 3 wavelengths were applied sequentially, first, infrared laser, followed by red laser, and then blue LED. The irradiation time of each laser device was manually controlled with a timer. The laser devices were calibrated automatically. The assessment tool was a plastic scale and a single assessor was used for measurements before and after LLLT.

Each laser device was administered on 10 points of each quadrant of the abdomen. The first point which was $3 \mathrm{~cm}$ apart from the umbilicus was chosen and then $3 \mathrm{~cm}$ apart laser device was used from one treatment point to another. Laser device was positioned at contact mode with a moderate pressure on tissue and titled at a $90^{\circ}$ angle. Time of irradiation was 15 seconds per point for each device $(1.5 \mathrm{~J} /$ point IR $+1.5 \mathrm{~J} /$ point red $+45 \mathrm{~J} /$ point the blue LED, in total $48 \mathrm{~J} \mathrm{IR}+\mathrm{RED}+$ Blue LED per point). Total energy was $480 \mathrm{~J} /$ quadrant and $1920 \mathrm{~J} /$ session. The umbilicus was used as an anamical landmark to differentiate parts of the abdomen for measurements. Girth of the abdomen at the site of umbilicus was noted as the middle abdomen, the girth of $4 \mathrm{~cm}$ above the umbilicus was measured as the upper abdomen and $4 \mathrm{~cm}$ and below the umbilicus was measured as the lower abdomen. The before and after evaluations were only made by measurement; and abdominal girths were measured before the beginning of LLLT and after completion of the therapy (Table 1).

Unfortunately, only few of the patients took part in the follow up measurement after 3 months, and as patients number was not enough, follow-up reports were skipped. No adverse events were reported during the study procedure. Some of the participants who suffered from constipation reported amelioration after 3 or 4 sessions of LLLT.

\section{Results}

A paired $t$ test was used to compare sizes of the upper, middle, and lower abdomen pre- to post-LLLT. Statistical analyses revealed that upper abdomen size significantly decreased from pre- $(91.86 \pm 11.16)$ to post-LLLT $(87.41$ \pm 10.52 $) \quad(P<0.001)$. Middle abdomen size showed significant reduction from pre- $(97.02 \pm 8.82)$ to postLLLT $(91.97 \pm 8.49)(P<0.001)$. Lower abdomen size significantly decreased from pre- $(100.36 \pm 9.45)$ to postLLLT $(95.80 \pm 8.52)(P<0.001)$ (Table 2).

Mean differences comparing the reduction in mean sizes of upper, middle and lower abdomen pre- to post-LLLT were $-4.81 \pm 2.31$ for upper abdomen, $-4.87 \pm 4.20$ for middle abdomen and $-4.12 \pm 1.66$ for lower abdomen (Table 3).

\section{Discussion}

The results of this study showed that LLLT using 3 different wavelengths of diode lasers is an effective and safe method for girth reduction.

Apparently, to assess the effectiveness of the combination of these wavelengths in comparison to each of them
Table 1. Abdominal Circumference Measurements Before and After LLLT

\begin{tabular}{|c|c|c|c|}
\hline No. of Patient & Age & $\begin{array}{l}\text { Size Before } \\
\text { LLLT }(\mathbf{c m})\end{array}$ & $\begin{array}{c}\text { Size After 12th } \\
\text { Session of LLLT }(\mathrm{cm})\end{array}$ \\
\hline 1 & 40 & $\begin{array}{c}U=80 \\
M=94 \\
L=100\end{array}$ & $\begin{array}{c}U=80 \\
M=87 \\
L=95\end{array}$ \\
\hline 2 & 50 & $\begin{array}{c}U=77 \\
M=84 \\
L=90\end{array}$ & $\begin{aligned} U & =74 \\
M & =79 \\
L & =88\end{aligned}$ \\
\hline 3 & 38 & $\begin{array}{c}U=103 \\
M=106 \\
L=110\end{array}$ & $\begin{array}{c}U=96 \\
M=98 \\
L=104\end{array}$ \\
\hline 4 & 38 & $\begin{array}{l}U=86 \\
M=95 \\
L=97\end{array}$ & $\begin{aligned} U & =80 \\
M & =90.5 \\
L & =95\end{aligned}$ \\
\hline 5 & 62 & $\begin{array}{c}U=91.5 \\
M=93 \\
L=95.5\end{array}$ & $\begin{aligned} U & =88 \\
M & =90.5 \\
L & =92\end{aligned}$ \\
\hline 6 & 32 & $\begin{array}{l}U=86 \\
M=92 \\
L=94\end{array}$ & $\begin{array}{c}U=84 \\
M=88 \\
L=90\end{array}$ \\
\hline 7 & 56 & $\begin{array}{c}U=92.5 \\
M=96 \\
L=98\end{array}$ & $\begin{array}{c}U=89 \\
M=92 \\
L=93\end{array}$ \\
\hline 8 & 50 & $\begin{array}{c}U=117 \\
M=120 \\
L=127\end{array}$ & $\begin{array}{c}U=114 \\
M=116 \\
L=122.5\end{array}$ \\
\hline 9 & 43 & $\begin{array}{l}U=92 \\
M=96 \\
L=103\end{array}$ & $\begin{array}{c}U=89 \\
M=91 \\
L=97\end{array}$ \\
\hline 10 & 45 & $\begin{array}{c}U=86 \\
M=88 \\
L=93\end{array}$ & $\begin{array}{c}U=82 \\
M=84 \\
L=89\end{array}$ \\
\hline 11 & 36 & $\begin{array}{c}U=88 \\
M=94 \\
L=95.5\end{array}$ & $\begin{array}{c}U=80 \\
M=91 \\
L=95.5\end{array}$ \\
\hline 12 & 62 & $\begin{array}{c}U=91.5 \\
M=93 \\
L=95.5\end{array}$ & $\begin{aligned} U & =88 \\
M & =90.5 \\
L & =92\end{aligned}$ \\
\hline 13 & 38 & $\begin{aligned} U & =80 \\
M & =90.5 \\
L & =95\end{aligned}$ & $\begin{array}{c}U=76 \\
M=85 \\
L=90\end{array}$ \\
\hline 14 & 43 & $\begin{array}{c}U=93 \\
M=102 \\
L=99\end{array}$ & $\begin{aligned} U & =83 \\
M & =93.5 \\
L & =95\end{aligned}$ \\
\hline 15 & 30 & $\begin{aligned} U & =98 \\
M & =100 \\
L & =97\end{aligned}$ & $\begin{array}{c}U=91.5 \\
M=93 \\
L=95\end{array}$ \\
\hline 16 & 27 & $\begin{array}{l}U=80 \\
M=88 \\
L=92\end{array}$ & $\begin{array}{c}U=78 \\
M=83 \\
L=87\end{array}$ \\
\hline 17 & 62 & $\begin{array}{c}U=115 \\
M=109 \\
L=110\end{array}$ & $\begin{aligned} U & =108 \\
M & =104 \\
L & =96\end{aligned}$ \\
\hline 18 & 51 & $\begin{array}{c}U=97 \\
M=106 \\
L=115\end{array}$ & $\begin{array}{c}U=93 \\
M=99.5 \\
L=108.5\end{array}$ \\
\hline
\end{tabular}


Table 2. Results of Paired T Test Comparing Sizes of Upper, Middle and Lower Abdomen Pre- to Post-LLLT

\begin{tabular}{lllll}
\hline & & Mean \pm SD & $t$ & $\boldsymbol{P}$ \\
\hline \multirow{2}{*}{ Upper abdomen } & Pre & $91.86 \pm 11.16$ & 7.60 & 0.00 \\
& Post & $87.41 \pm 10.52$ & & \\
\multirow{2}{*}{ Middle abdomen } & Pre & $97.02 \pm 8.82$ & 12.21 & 0.00 \\
& Post & $91.97 \pm 8.49$ & & \\
Lower abdomen & Pre & $100.36 \pm 9.45$ & 6.70 & 0.00 \\
& Post & $95.80 \pm 8.52$ & & \\
\hline
\end{tabular}

Table 3. Mean Differences Comparing the Reduction in Mean Sizes of Upper, Middle and Lower Abdomen Pre- to Post-LLLT

\begin{tabular}{lc}
\hline & Mean Differences From Pre- to Post-LLLT $(\mathbf{c m})$ \\
\hline Upper abdomen & $-4.81 \pm 2.31$ \\
Middle abdomen & $-4.87 \pm 4.20$ \\
Lower abdomen & $-4.12 \pm 1.66$ \\
\hline
\end{tabular}

alone, there should have been at least 5 study groups: red laser alone, infrared laser alone, blue LED alone, red and infrared, and the combination of the 3 wavelengths. This research was undertaken with a before-after case series as a pilot study. It was accepted that this case series could not be used as a clinical reference. A multi-groups clinical trial is the next plan for further investigation on this category.

\section{Conclusion}

LLLT is a safe, non-invasive and pain free technique, which is effective in reducing local adiposity. Based on this case series pilot investigation, combination of 3 different wavelengths of LLL was effective for abdominal girth reduction in $100 \%$ of our subjects $(P<0.001)$, without any side effects. But it was accepted that there is a need for large sample size and placebo/control groups. The next step is to investigate this combination of wavelengths in a clinical case/control groups study.

\section{Ethical Considerations}

All subjects signed the informed consent form in accordance with the Declaration of Helsinki and satisfied all the study eligibility criteria.

\section{Conflict of Interests}

The authors have no conflict of interest to declare.

\section{References}

1. Rossi AB, Vergnanini AL. Cellulite: a review. J Eur Acad Dermatol Venereol. 2000;14(4):251-262. doi:10.1046/ j.1468-3083.2000.00016.x.

2. Pinna K. Nutrition and Diet Therapy. Belmont, CA: Wadsworth; 2007:178

3. Emanuele E, Bertona M, Geroldi D. A multilocus candidate approach identifies ACE and HIF1A as susceptibility genes for cellulite. J Eur Acad Dermatol Venereol. 2010;24 (8):930935. doi:10.1111/j.1468-3083.2009.03556.x.

4. Jackson RF, Roche GC, Shanks SC. Double-blind, placebocontrolled randomized trial "evaluating the ability of low- level laser therapy to improve the appearance of cellulite." Lasers Surg Med. 2013;45(3):141-147.

5. Wanner M, Avram M. An evidence-based assessment of treatments for cellulite. J Drugs Dermatol. 2008;7(4):341345.

6. Pavicic T, Borelli C, Korting HC. Cellulite--the greatest skin problem in healthy people? An approach. J Dtsch Dermatol Ges. 2006;4(10):861-870. doi:10.1111/j.16100387.2006.06041.x.

7. Terranova F, Berardesca E, Maibach H. Cellulite:nature and aetiopathogenesis. Int J Cosmet Sci. 2006;28(3):157-167. doi:10.1111/j.1467-2494.2006.00316.x.

8. Mulholland RS, Paul M, Chalfoun C. Noninvasive body contouring with radiofrequency, ultrasound, cryo-lipolysis, and low-level laser therapy. Clin Plastic Surg. 2011;38:503520.

9. Maloney R, Shanks S, Jenney E. The reduction in cholesterol and triglyceride serum levels following low-level laser irradiation:a non-controlled, non-randomized pilot study. Laser Surg Med. 2009;21S:66.

10. Avci P, Nyame TT, Gupta GK, Sadasivam M, Hamblin MR. Low-level laser therapy for fat layer reduction: a comprehensive review. Laser Surg Med. 2013;45(6);349357. doi:10.1002/lsm.22153.

11. Jackson FR, Roche GC, Wisler K. Reduction in cholesterol and triglycerides serum levels following low level laser irradiation: a noncontrolled, nonrandomized pilot study. Am J Cosmet Surg. 2010;27(4):177-184.

12. Rushdi TA. Effect of low-level laser therapy on cholesterol and triglyceride levels in ICU patients: a controlled randomized study. EJCTA. 2010;4:96-99.

13. Karu TI. Mitochondrial signaling in mammalian cells activated by red and near-IR radiation. Photochem Photobiol. 2008;84:1091-1099.

14. Karu TI. Photobiology of low-power laser effects. Health Phys. 1989;56:691-704.

15. Karu TI, Afanasyeva NI. Cytochrome C oxidase as primary photo acceptor for cultured cells in visible and near IR regions. DokladyAkad Nauk (Moscow). 1995;342:693-695.

16. Rosenbaum M, Prieto V, Hellmer J, et al. An exploratory investigation of the morphology and biochemistry of cellulite. Plast Reconstr Surg. 1998;101:1934-1939.

17. Lach E, Pap S. Laser treatment for cellulite:A noninvasive alternative to liposuction. Lasers Surg Med. 2004;34:S16-S32.

18. Jackson RF, Dedo DD, Roche GC, Turok DI, Maloney RJ. Low-level laser therapy as a non-invasive approach for body contouring:a randomized, controlled study. Lasers Surg Med. 2009;41:799-809.

19. Savoia A, Landi S, Vannini F, Baldi A. Low-level laser therapy and vibration therapy for the treatment of localized adiposity and fibrous cellulite. Dermatol Ther (Heidelb). 2013;3(1):41-52.

20. Nestor MS, Newburger J, Zarraga MB. Body contouring using 635-nm low level laser therapy. Semin Cutan Med Surg. 2013;32:35-40.

21. Nestor MS, Zarraga MB, Park H. Effect of $635 \mathrm{~nm}$ lowlevel laser therapy on upper arm circumference reduction: a double-blind, randomized, sham-controlled trial. J Clin Aesthet Dermatol. 2012;5:42-48.

22. McRae E, Boris J. Independent evaluation of low-level laser therapy at $635 \mathrm{~nm}$ for non-invasive body contouring of the waist, hips, and thighs. Lasers Surg Med. 2013;45(1):1-7. 
23. Neira R, Arroyave J, Ramirez H, et al. Fat liquefaction: effect of low level laser energy on adipose tissue. Plast Reconstr Surg. 2002;110:912-922. doi:10.1097/00006534200209010-00030.

24. Lucassen GW, van der Sluys WL, van Herk JJ, et al. The effectiveness of massage treatment on cellulite as monitored by ultrasound imaging. Skin Res Technol. 1999;3:154-160.

25. Kolari PJ. Penetration of unfocused laser light into the skin. Arch Dermatol Res. 1985;277:342-344. doi:10.1007/ BF00509097.

26. Kolárová $\mathrm{H}$, Ditrichova $\mathrm{D}$, Wagner J. Penetration of the laser light into the skin in vitro. Lasers Surg Med. 1999;24:231235. doi:10.1002/(SICI)1096-9101(1999)24:3<231::AIDLSM8>3.0.CO;2-\#.

27. Enwemeka CS. Attenuation and penetration of visible 632.8 $\mathrm{nm}$ and invisible infra-red $904 \mathrm{~nm}$ light in soft tissues. Laser Ther J. 2003;13:16.

28. Smith AM, Mancini MC, Nie S. Bioimaging: second window for in vivo imaging. Nat Nanotechnol. 2009;4(11):710-711. doi: 10.1038/nnano.2009.326

29. Furchgott RF, Ehrreich SJ. Relaxation of mammalian smooth muscles by visible and ultraviolet radiation. Nature. 1968;218(5142):682-684.

30. Mittermayr R, Osipov A, Piskernik C, et al. Blue Laser Light increases perfusion of a skin flap via release of nitric oxide from hemoglobin. Mol Med. 2007;13(1-2):22-9. doi:10.2119/2006-00035.

31. Kozlov AV, Costantino G, Sobhian B, et al. Mechanisms of vasodi-latation induced by nitrite instillation in intestinal lumen:possible role of hemoglobin. Antioxid Redox Signal. 2005; 7:515-721

32. Gladwin MT, Crawford JH, Patel RP. The biochemistry of nitric oxide, nitrite, and hemoglobin:role in blood flow regulation. Free Radic Biol Med. 2004;36:707-717.

33. Cosby K, Partovi KS, Crawford JH, et al. Nitrite reduction to nitric oxide by deoxyhemoglobin vasodilates the human circulation. Nat Med. 2003;9:1498-1505. 